\title{
12 Weeks of Combined Endurance and Resistance Training Reduces Innate Markers of Inflammation in a Randomized Controlled Clinical Trial in Patients with Multiple Sclerosis
}

\author{
Nathalie Deckx, ${ }^{1}$ Inez Wens, ${ }^{2}$ Amber H. Nuyts, ${ }^{1}$ Niel Hens, ${ }^{3,4}$ \\ Benedicte Y. De Winter, ${ }^{5}$ Gudrun Koppen, ${ }^{6}$ Herman Goossens, ${ }^{7}$ Pierre Van Damme, \\ Zwi N. Berneman, ${ }^{1}$ Bert O. Eijnde, ${ }^{2}$ and Nathalie Cools ${ }^{1}$ \\ ${ }^{1}$ Laboratory of Experimental Hematology, Vaccine \& Infectious Disease Institute (VAXINFECTIO), \\ Faculty of Medicine and Health Sciences, University of Antwerp, 2610 Wilrijk, Belgium \\ ${ }^{2}$ REVAL Rehabilitation Research Centre, BIOMED Biomedical Research Institute, Faculty of Medicine and Life Sciences, \\ Hasselt University, 3590 Diepenbeek, Belgium \\ ${ }^{3}$ Interuniversity Institute for Biostatistics and Statistical Bioinformatics (I-BIOSTAT), Hasselt University, 3590 Diepenbeek, Belgium \\ ${ }^{4}$ Centre for Health Economic Research and Modelling Infectious Diseases (CHERMID), Vaccine \& \\ Infectious Disease Institute (VAXINFECTIO), Faculty of Medicine and Health Sciences, University of Antwerp, 2610 Wilrijk, Belgium \\ ${ }^{5}$ Laboratory of Experimental Medicine and Pediatrics, Division of Gastroenterology, \\ Faculty of Medicine and Health Sciences, University of Antwerp, 2610 Wilrijk, Belgium \\ ${ }^{6}$ Flemish Institute for Technological Research (VITO), Environmental Risk and Health Unit, 2400 Mol, Belgium \\ ${ }^{7}$ Laboratory of Medical Microbiology, Vaccine \& Infectious Diseases Institute (VAXINFECTIO), \\ Faculty of Medicine and Health Sciences, University of Antwerp, 2610 Wilrijk, Belgium \\ ${ }^{8}$ Centre for the Evaluation of Vaccination, Vaccine \& Infectious Disease Institute (VAXINFECTIO), \\ Faculty of Medicine and Health Sciences, University of Antwerp, 2610 Wilrijk, Belgium
}

Correspondence should be addressed to Nathalie Cools; nathalie.cools@uza.be

Received 31 August 2015; Revised 30 November 2015; Accepted 7 December 2015

Academic Editor: Teresa Zelante

Copyright (c) 2016 Nathalie Deckx et al. This is an open access article distributed under the Creative Commons Attribution License, which permits unrestricted use, distribution, and reproduction in any medium, provided the original work is properly cited.

Previously, we reported that patients with multiple sclerosis (MS) demonstrate improved muscle strength, exercise tolerance, and lean tissue mass following a combined endurance and resistance exercise program. However, the effect of exercise on the underlying disease pathogenesis remains elusive. Since recent evidence supports a crucial role of dendritic cells (DC) in the pathogenesis of MS, we investigated the effect of a 12 -week combined exercise program in MS patients on the number and function of DC. We demonstrate an increased number of plasmacytoid DC (pDC) following the exercise program. These pDC display an activated phenotype, as evidenced by increased numbers of circulating CD62 $\mathrm{L}^{+}$and $\mathrm{CD} 80^{+} \mathrm{pDC}$. Interestingly, the number of CD80 ${ }^{+} \mathrm{pDC}$ positively correlates with the presence of IL-10-producing regulatory type 1 cells (Tr1), an important cell type for maintaining peripheral tolerance to self-antigens. In addition, decreased production of the inflammatory mediators, TNF- $\alpha$ and MMP-9, upon Toll-like receptor (TLR) stimulation was found at the end of the exercise program. Overall, our findings suggest that the 12-week exercise program reduces the secretion of inflammatory mediators upon TLR stimulation and promotes the immunoregulatory function of circulating pDC, suggestive for a favorable impact of exercise on the underlying immunopathogenesis of MS.

\section{Introduction}

Multiple sclerosis (MS) is a chronic demyelinating, inflammatory disease of the central nervous system (CNS), predominantly affecting young adults in their most productive years.
It is a heterogeneous disease with regard to both its clinical course and symptoms. The most prevalent symptoms are sensory changes, including fatigue, balance disorders, spasticity, motor weakness, and impaired muscular performance [1]. Besides incremental disability, these symptoms also result 
in a reduced quality of life. Nevertheless, a considerable part of MS symptoms may also result from a sedentary lifestyle [2]. Over the years, different studies have reported improvement in muscle strength [3], cardiovascular health [4], mental functioning [2], and quality of life [5] following a physical exercise intervention in MS patients. Recently, we demonstrated improvement in muscle strength, exercise tolerance, and lean tissue mass in MS patients following a combined endurance and resistance exercise program [6]. Although it is likely that only the effects of a sedentary lifestyle can be reversed by physical exercise [7], it has been suggested that physical exercise might have the potential to have an impact on the disease pathogenesis of MS as well [8]. To date, however, little is known about the potential effects of physical exercise on the underlying disease mechanisms.

Although the exact cause of MS is still unknown, it is now generally accepted that MS is a complex multifactorial disease involving genetic and environmental factors affecting the autoreactive immune response [9]. Based on previous efforts focusing on the role of the adaptive immune system in the pathogenesis of MS, it is currently well established that autoreactive Thelper type 1 (Th1) and Th17 cells mediate the inflammatory processes in the CNS [10]. Recent evidence also suggests involvement of innate immunity, including dendritic cells (DC), in the initiation and maintenance as well as progression of MS [11].

DC are professional antigen-presenting cells with the unique capacity to polarize the differentiation of $\mathrm{T}$ cells, thereby regulating the balance between inflammation and tolerance [12]. To perform this function, DC are capable of capturing antigens, processing them, and presenting them on the cell surface complexed to major histocompatibility (MHC) molecules, for example, human leukocyte antigen(HLA-) DR. Along with that, context-dependent expression of costimulatory molecules, such as CD80 and CD86, and secretion of cytokines occurs. In human blood, two major subsets of DC have been characterized, that is, myeloid or conventional DC (cDC) and plasmacytoid DC (pDC) [13]. Both DC subsets display a different expression profile of cytokine receptors and cytokines, of migratory markers and migration potential, and of Toll-like receptors (TLR). In brief, cDC strongly express TLR2 and TLR4, among other TLR, which are stimulated by bacterial triacylated lipopeptides, Gram-negative bacterial lipopolysaccharide (LPS), fungal mannans, parasitic phospholipids, viral envelope proteins, and host-derived heat shock proteins. Upon activation, cDC secrete proinflammatory cytokines such as interleukin- (IL-) $1 \beta$, IL-6, IL-12p70, and tumour necrosis factor- (TNF-) $\alpha$ [13]. In addition, $\mathrm{CDC}$ also secrete the matrix metalloproteinase(MMP-) 9, a protease that is involved in the breakdown of extracellular matrix in normal physiological processes, including cell migration [14]. On the other hand, pDC express TLR7 and TLR9, which are stimulated by single-stranded RNA and unmethylated CpG oligodeoxynucleotides. Upon activation, pDC secrete significant amounts of type I interferon (IFN) [15]. Depending on their costimulatory molecule expression and cytokine secretion, both $\mathrm{cDC}$ and $\mathrm{pDC}$ are able to induce effector $\mathrm{T}$ cells as well as regulatory
T cells (Treg) [12]. While in a steady state, both $\mathrm{cDC}$ and $\mathrm{pDC}$ are poor $\mathrm{T}$ cell stimulators, given their low expression levels of MHC and costimulatory molecules, activated $\mathrm{cDC}$ and pDC differ strongly in their T cell-stimulatory capacity. Following TLR ligation, activated cDC efficiently stimulate effector $\mathrm{T}$ cells as they upregulate the expression of costimulatory molecules and cytokines. On the other hand, TLR-mediated recognition of viral products and RNA/ DNA/immunocomplexes by pDC results in the production of large amounts of type I IFN [15]. For this, pDC effectively participate in antiviral immunity. Nevertheless, besides this protective role, $\mathrm{pDC}$ may also play a role in aberrant immune responses. For instance, it has been suggested that pDC promote the progression of systemic lupus erythematosus following sensing of self-DNA and subsequent production of type I IFN [16]. In contrast, pDC have been implicated in maintenance of tolerance. It has been reported that pDC contribute to peripheral $\mathrm{T}$ cell tolerance in transplantation [17], tumour escape [18], oral tolerance [19], and mucosal tolerance [20]. Indeed, pDC display tolerogenic functions with the ability to indirectly suppress $\mathrm{T}$ cell proliferation through the induction of Treg [21].

Because of their unique capacity to shape immune responses [12], DC are thought to play a pivotal role in the immunopathogenesis of several autoimmune disorders, including MS. Previously, we reported an aberrant frequency and expression of costimulatory markers on DC in the peripheral blood of MS patients. Circulating DC also display divergent expression of migration markers in MS patients as compared to DC from healthy controls. In addition, we demonstrated increased secretion of IL-12p70 by LPSand IFN- $\gamma$-stimulated DC from relapsing-remitting (RR) MS patients as compared to healthy controls [22]. Furthermore, upon stimulation with TLR9 ligands, IFN $\alpha$ secretion by pDC from RRMS patients is reduced as compared to the level secreted by pDC from healthy controls [23].

Previously, others demonstrated that physical exercise alters the number and function of leukocytes, including circulating cells of the innate immune system, such as neutrophils, monocytes, and natural killer cells [24]. However, the effect of physical exercise on DC is rather unexplored. Increased DC numbers were found following exercise training in an animal model, whereas their phenotype, that is, the expression of the costimulatory molecules CD80 and CD86 by DC, was not affected [25]. Interestingly, after 5 weeks of physical exercise, bone marrow-derived rat DC displayed a more pronounced immune-stimulatory function [25]. Also in humans, increased numbers of $\mathrm{cDC}$, but not $\mathrm{pDC}$, were found following exercise [26, 27].

Altogether, it has been demonstrated that physical exercise is well tolerated and induces relevant improvements in both physical and mental functioning of MS patients, although its effect on underlying mechanisms remains to be elucidated. Since recent evidence suggests a crucial role for innate immunity in the initiation as well as progression of MS, we aim to investigate the effect of a 12-week combined endurance and resistance exercise program on cellular and molecular mediators of innate immunity in MS patients. 


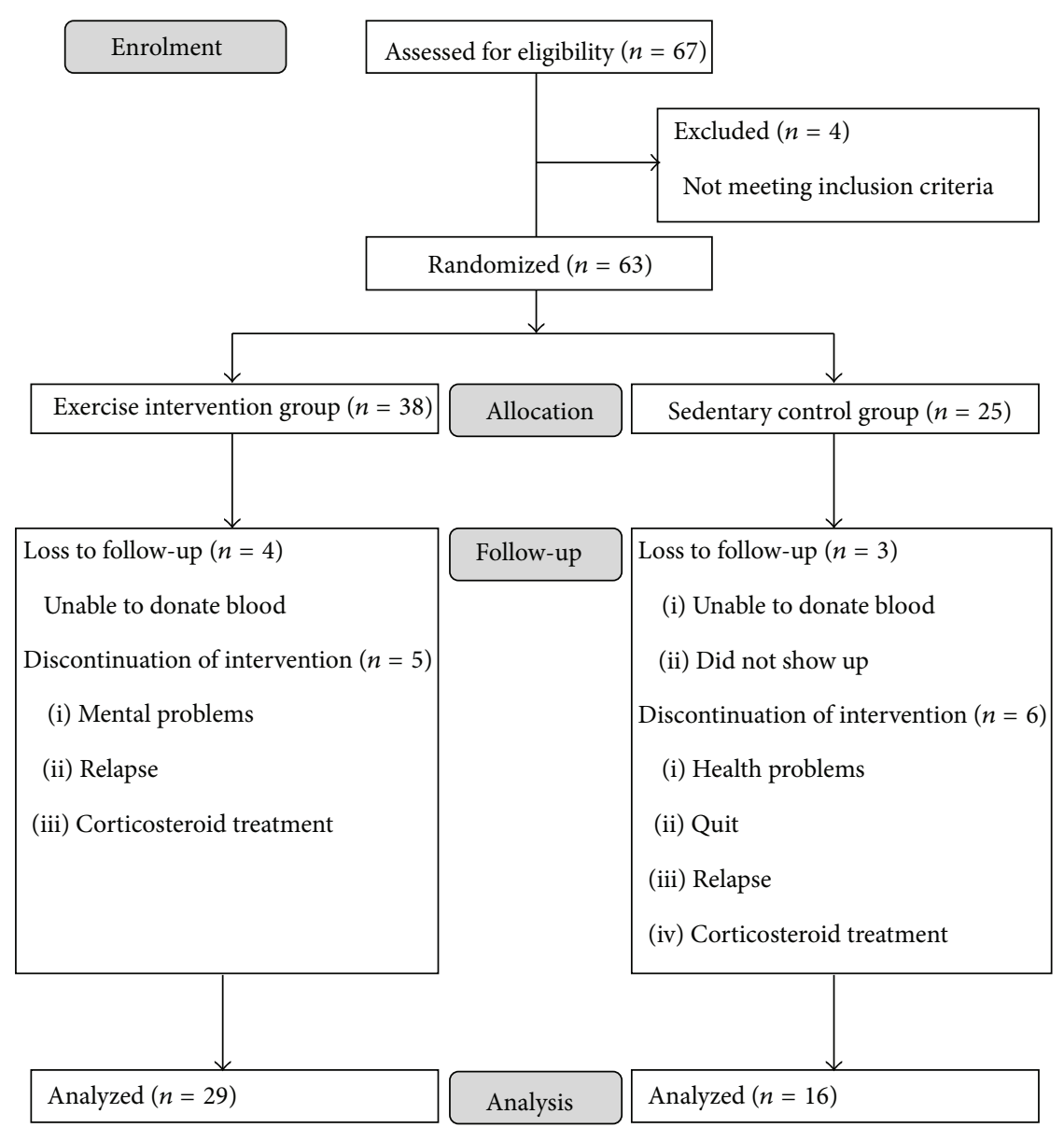

FIGURE 1: Flow diagram of patient enrolment.

\section{Material and methods}

2.1. Study Population. A total of 67 MS patients, diagnosed according to the revised McDonald criteria [28] and aged $>18$ years, were assessed for eligibility by the REVAL Rehabilitation Research Centre. Subjects were excluded if they had an expanded disability status scale (EDSS) score $>6$, that is, not able to walk $100 \mathrm{~m}$ without walking aid, physician-diagnosed diabetes mellitus type II, other autoimmune diseases (diabetes mellitus type I and/or rheumatoid arthritis), or other chronic diseases (cardiovascular, pulmonary, and/or renal), were pregnant, participated in another study, had contraindications to perform physical exercise, received corticosteroid treatment 3 months prior to the study, or had an acute MS exacerbation 6 months prior to the study. All subjects gave informed consent in accordance with the declaration of Helsinki and the protocol was approved by the local Ethics Committee of Virga Jesse Hospital, Hasselt, and Hasselt University and registered at https://www.clinicaltrials.gov/ (NCT01718392 and NCT01845896).

2.2. Study Design. MS patients were randomized by sealed envelope following a $3: 2$ ratio to an exercise intervention group (EX: $n=38$ ) or a sedentary control group (SED: $n=25)$ by the REVAL Rehabilitation Research Centre.
Respectively, only 29 and 16 patients were included in the repeated measures analysis, due to loss to follow-up or discontinuation of intervention (Figure 1). The ratio of patients who discontinued the intervention and the ratio of patients who were lost to follow-up were not significantly different between the exercise and the sedentary group (see Supplementary Appendix in Supplementary Material available online at http://dx.doi.org/10.1155/2016/6789276).

Patient characteristics and medication use are depicted in Supplementary Table 1. Due to the nature of the trial, patients and caregivers could not be blinded to group allocation, whereas those assessing the outcomes were blinded to group allocation. Patients in the exercise group participated in a supervised 12-week combined endurance and resistance training program in the REVAL Rehabilitation Research Centre at a frequency of 5 sessions per 2 weeks. Each session started with a cardiovascular part, consisting of cycling and treadmill walking or running (Technogym, LJ Capelle aan den Ijssel, The Netherlands). Session duration and intensity increased as the program proceeded, starting from $1 \times 6 \mathrm{~min} /$ session to $3 \times 10 \mathrm{~min} /$ session, interspersed by 2-3 minutes of rest. The duration was determined beforehand, whereas the intensity was determined individually depending on heart rate and disability level. For example, participants needed to be able to walk/pedal uninterrupted 
and independently for the prescribed minutes, and intensity decreased when they had difficulties keeping up with the pace or felt not confident to succeed in the program. The second part consisted of resistance training (leg press, leg curl, leg extension, vertical traction, arm curl, and chest press, Technogym). Resistance training of the lower limb was performed unilaterally, due to bilateral strength differences between the legs of MS patients. To improve muscle fitness, sets of repetitions gradually increased during intervention, from $1 \times 10$ repetitions to $4 \times 15$ repetitions, with maximal attainable load, interspersed by 1-2 minutes of rest. The number of repetitions was determined beforehand, whereas the intensity was determined individually, based on individual disability levels, which are inconstant in MS. When the number of repetitions increased, the maximal attainable load decreased. After two or three sessions, working with the same number of repetitions, participants were encouraged to, if possible, increase their weights again. Continuous encouragement and supervision by the instructors during the training period led to a systematic increase of the training load over the 12-week training period. The sessions were finished by stretching. Patients in the sedentary group did not participate in any training program and were asked to continue standard care. Blood $(20 \mathrm{~mL})$ was sampled from patients in both groups in fasting state at 8 a.m. following abstinence from exercise for 48 hours. Samples were collected before the combined exercise program (PRE) and within one week after the end of the 12-week training program (POST) in both heparin and serum-separating tubes (BD Biosciences, Erembodegem, Belgium).

2.3. Isolation and Stimulation of Leukocytes. Leukocyte cell counts were measured using an automated cell counter (ABX Micros 60, Horiba, Deurne, Belgium). Next, leukocytes were isolated for ex vivo flow cytometric analysis of DC and Treg subsets using density gradient purification (Ficoll Paque PLUS, GE Healthcare, Chalfont St Giles, UK). In addition, for evaluation of Treg frequencies using intracellular flow cytometry, leukocytes were treated overnight with brefeldin A $(10 \mu \mathrm{g} / \mathrm{mL})$ (Life Technologies, Merelbeke, Belgium). Simultaneously, $1 \mathrm{~mL}$ of peripheral blood was (i) stimulated overnight with $2 \mu \mathrm{g} / \mathrm{mL}$ LPS, a TLR4 ligand (Invivogen, Toulouse, France), and $50 \mu \mathrm{g} / \mathrm{mL}$ IFN$\gamma$ (Immunotools, Friesoythe, Germany) or (ii) stimulated overnight with $10 \mu \mathrm{g} / \mathrm{mL}$ imiquimod (IQ), a synthetic TLR7 ligand (Invivogen) or (iii) left untreated as a control. For evaluation of TLR-mediated cytokine production, plasma was collected and stored at $-20^{\circ} \mathrm{C}$ for further use in cytokine secretion assays. For evaluation of DC activation state, leukocytes were enriched after red blood cell lysis $\left(0.155 \mathrm{M} \mathrm{NH}_{4} \mathrm{Cl}\right.$, $0.01 \mathrm{M} \mathrm{KHCO}_{3}$, and $0.1 \mathrm{mM} \mathrm{Na}_{2}$-EDTA).

2.4. Flow Cytometry. Immunophenotyping of DC was done by direct immunofluorescence staining using the following fluorochrome-labeled mouse anti-human monoclonal antibodies: anti-blood dendritic cell antigen- (BDCA-) 1 phycoerythrin (PE) (Miltenyi Biotec, Leiden, The Netherlands), anti-BDCA-2 allophycocyanin (APC) (Miltenyi Biotec), anti-lineage I (Lin I; anti-CD3, anti-CD14, anti-CD16, antiCD19, anti-CD20, and anti-CD56) fluorescein isothiocyanate (FITC) (BD Biosciences), anti-CD62L PE-cyanine 7 (PECy7) (eBioscience, Vienna, Austria), anti-CD80 APC-H7 (BD Biosciences), anti-CD86 V450 (BD Biosciences), antiCCR5 PE-Cy7 (BD Biosciences), anti-CCR7 V450 (BD Biosciences), and anti-human leukocyte antigen- (HLA-) DR APC-H7 (BD Biosciences) antibodies. Moreover, dead cells were excluded by addition of violet live/dead stain (Life Technologies) to the antibody mixture. $\mathrm{cDC}$ and $\mathrm{pDC}$ were identified as $\mathrm{Lin} \mathrm{I}^{-} \mathrm{BDCA}_{-}{ }^{+}$and $\mathrm{Lin} \mathrm{I}^{-} \mathrm{BDCA}_{-}{ }^{+}$, respectively. The number of DC displaying an activated or a migratory phenotype was assessed by enumerating $\mathrm{CDC}$ and pDC expressing costimulatory molecules (CD80, CD86) and migration markers (CD62L, CCR5, and CCR7), respectively.

Treg subsets were characterized using the following fluorochrome-labeled mouse anti-human monoclonal antibodies: anti-CD3 peridinin chlorophyll protein Cy5.5 (PerCP-Cy5.5) (BD Biosciences), anti-CD4 APC-H7 (BD Biosciences), anti-CD8 pacific blue (PB) (Life Technologies), anti-CD25 PE-Cy7 (BD Biosciences), anti-IL-10 APC (BD Biosciences), anti-transforming growth factor- (TGF-) $\beta$ PE (IQ Products, Groningen, The Netherlands), and antiforkhead box P3 (FoxP3) Alexa Fluor 488 (BD Biosciences). Moreover, dead cells were excluded by addition of violet live/dead stain (Life Technologies) to the antibody mixture. Naturally occurring Treg were identified as CD2 $5^{\text {hi }}$ FoxP3 ${ }^{+}$, while induced Treg, that is, regulatory type 1 cells (Tr1) and $\mathrm{T}$ helper 3 (Th3) cells, were characterized based on the production of the anti-inflammatory cytokines IL-10 and TGF- $\beta$, respectively.

Fluorescence minus one in combination with nonreactive isotype-matched antibodies was used as control. For analytical flow cytometry, at least $10^{5}$ events were measured using a three-laser instrument configured to measure up to 15 parameters simultaneously, that is, a Cyflow ML flow cytometer (Partec, Münster, Germany). All results were analyzed using FlowJo software (Tree Star, Inc., Ashland, OR, USA).

2.5. Soluble Analyte Secretion Assays. Serum was isolated using serum-separating tubes (BD Biosciences). Quantitative detection of cortisol (Alpco, Salem, NH, USA) and dehydroepiandrosterone (DHEA; Alpco), was determined by means of ELISA according to manufacturer's instructions. Levels of the primary human anti-inflammatory glucocorticoid, cortisol, are often insufficient to evaluate the function of the hypothalamus-pituitary-adrenal (HPA) axis. Therefore, we also quantified the serum concentration of DHEA, a precursor of the sex hormones testosterone and estrogen and a glucocorticoid antagonist, in order to calculate the molar ratio of cortisol to DHEA [29, 30]. In addition, serum levels of IL-6, IL-10, IL-12p70, TNF- $\alpha$, MMP-9, and the inflammasome effector molecule caspase1 (R\&D, Minneapolis, MN, USA) were quantified using ELISA according to manufacturer's instructions. All kits were purchased from Meso Scale Discovery (MSD, Rockville, MD, USA), unless stated otherwise. For quantitative detection of 
the cytokines secreted following stimulation of peripheral blood, collected plasma samples were analyzed using the following commercially available ELISA kits: IL-1 $\beta$, IL-6, IL12 p70, TNF- $\alpha$, IFN- $\alpha$ (PBL InterferonSource, Piscataway, NJ, USA), caspase-1 (R\&D), and MMP-9, according to manufacturer's instructions. All kits were purchased from eBioscience, unless stated otherwise.

2.6. Statistical Analysis. All data were analyzed using SAS 9.3 software (SAS Institute Inc., Cary, USA). Linear mixed models were used to analyze repeated measures data [31]. A model was built stepwise per outcome variable, starting from a univariate model with "time" as the only fixed effect. Step by step new models were constructed by adding other fixed effect variables including group, MS type, gender, age, body mass index (BMI), EDSS, and MS-specific medication. The variable "group" included trained (EX) and sedentary MS patients (SED). The variable "MS type" included relapsingremitting (RR) and chronic-progressive (CP) MS patients. The variable "MS-specific medication" included untreated 1st-line treatment and 2nd-line treatment. A $P$ value $<0.10$ ( $F$ test) was used as the threshold for retaining a fixed effect during model building to decrease the chance of missing a significant effect in the final model. Subsequently, possible interaction effects between the retained fixed effects were assessed and also retained if $P<0.10$. For interpretation of the fixed effects in the final model, the threshold for statistical significance was set at $P<0.05$. Since we aim to focus on temporal effects, we only report main and interaction effects of time. Furthermore, since our main interest is the effect of time, we did not include baseline measurements of patients who did not complete the study, that is, patients who were lost to follow-up or patients who discontinued the intervention. No bias was expected for excluding baseline measurements from patients who did not complete the study (Supplementary Appendix). Nevertheless, to determine safety of the intervention, patients who relapsed during the study were noted and the annualized relapse rate was calculated for both groups. Models consisting of more than 12 parameters were not interpreted because of the risk of overfitting. With significant interaction effects of time, post hoc analyses were performed using the Bonferroni correction to correct for multiple testing. Moreover, based on our a priori hypotheses that an effect of time, that is, the 12week exercise intervention, could be observed in the trained group of patients and not in the sedentary group of patients [24], post hoc analyses for evaluating the effect of time in both the trained and the sedentary group were always performed, thereby reducing the chance of false negative results. Moreover, we used the conservative Bonferroni correction to account for multiple testing, thereby reducing the chance of detecting false positive results (Supplementary Appendix). Diagnostics were based on the studentized residuals and response variables were log-transformed when necessary. Finally, relevant correlations were assessed using Pearson's correlation test. Graphs were generated in SAS 9.3 software and GraphPad version 5 software (Prism, La Jolla, CA, USA). All data are presented as mean \pm standard error of the mean (SEM).
TABLE 1: Clinical details of the study population.

\begin{tabular}{lccc}
\hline & EX & SED & $P$ values \\
\hline Gender (M/F) & $13 / 16$ & $6 / 10$ & 0.634 \\
Age \pm SEM & $47 \pm 2$ & $50 \pm 3$ & 0.516 \\
BMI \pm SEM & $24 \pm 1$ & $24 \pm 1$ & 0.821 \\
EDSS \pm SEM & $3 \pm 0.2$ & $3 \pm 0.4$ & 0.155 \\
Type MS (CP/RR) & $10 / 19$ & $5 / 11$ & 0.826 \\
$\begin{array}{l}\text { Medication } \\
\text { (untreated/1st-line }\end{array}$ & & & \\
treatment/2nd-line & $4 / 16 / 9$ & $2 / 8 / 6$ & 0.908 \\
treatment) & & & \\
\hline
\end{tabular}

Results are shown as mean \pm SEM. Patients were defined as untreated when a wash-out period of at least 3 months was respected before recruitment in the study. 1st-line treatment: IFN- $\beta$ (Avonex, Betaferon, and Rebif) and glatiramer acetate (Copaxone); 2nd-line treatment: alemtuzumab (Campath), natalizumab (Tysabri), and fingolimod (Gilenya).

EX, exercise group; SED, sedentary control group; M, male; F, female; BMI, body mass index; EDSS, expanded disability status scale; MS, multiple sclerosis; CP, chronically progressive MS; RR, relapsing-remitting MS; SEM, standard error of the mean.

\section{Results}

3.1. No Baseline Differences between Trained and Sedentary MS Patients. MS patients were randomized following a 3:2 ratio to an exercise intervention group (EX: $n=38$ ) or a sedentary control group (SED: $n=25$ ). Patients in the exercise group reached a mean adherence percentage of $90 \%$, ranging from 80 to $100 \%$, at the end of the 12 -week training program. At the end of the study, all data points from 29 and 16 patients in the exercise and the sedentary group, respectively, were collected for analysis (Figure 1), changing the ratio to $2: 1$. No differences in the distribution of gender, age, BMI, clinical course of MS, EDSS, and use of MS-specific medication could be detected for the analyzed patients between both study groups (Table 1). Similarly, no differences at baseline could be detected for all experimental outcomes tested between the exercise group and the sedentary control group except for the number of $\mathrm{CCR}^{+} \mathrm{CDC}(P=0.033)$, which was higher in the exercise group (vide infra).

\subsection{Serum Signaling Molecule Concentrations Are Not Altered} following Long-Term Physical Exercise in MS. Although no difference in the cortisol concentration between the patient groups in the course of the 12-week time period could be detected, cortisol levels significantly increased in the trained group after the 12-week combined exercise program $(P=$ 0.047; Table 2), while no change was observed in the sedentary control group. However, the physiological relevance of this relatively small increase (fold increase $=1.07$ ) is debatable. No significant differences of the concentration of DHEA and the molar concentration of cortisol to DHEA could be detected following the 12-week time period between the exercise group and the sedentary control group. In our hands, no significant differences could be detected between the exercise group and the sedentary control group in the course of the 12-week time period with regard to the serum 
TABLE 2: Serum signaling molecule concentrations are not altered following long-term physical exercise in MS.

\begin{tabular}{|c|c|c|c|c|}
\hline Molecule & Group & Time & Mean concentration $(\mathrm{pg} / \mathrm{mL}) \pm$ SEM & $P$ values \\
\hline \multirow{4}{*}{ IL-6 } & \multirow{2}{*}{ EX } & PRE & $0.60 \pm 0.09$ & \multirow{2}{*}{0.434} \\
\hline & & POST & $0.54 \pm 0.09$ & \\
\hline & \multirow{2}{*}{ SED } & PRE & $0.45 \pm 0.09$ & \multirow{2}{*}{0.341} \\
\hline & & POST & $0.54 \pm 0.09$ & \\
\hline \multirow{4}{*}{ IL-10 } & \multirow{2}{*}{ EX } & PRE & $0.48 \pm 0.09$ & \multirow{2}{*}{0.731} \\
\hline & & POST & $0.50 \pm 0.09$ & \\
\hline & \multirow{2}{*}{ SED } & PRE & $0.60 \pm 0.19$ & \multirow{2}{*}{0.097} \\
\hline & & POST & $0.31 \pm 0.04$ & \\
\hline \multirow{4}{*}{ IL-12p70 } & \multirow{2}{*}{ EX } & PRE & $0.09 \pm 0.01$ & \multirow{2}{*}{0.312} \\
\hline & & POST & $0.12 \pm 0.02$ & \\
\hline & \multirow{2}{*}{ SED } & PRE & $0.13 \pm 0.03$ & \multirow{2}{*}{1.000} \\
\hline & & POST & $0.13 \pm 0.02$ & \\
\hline \multirow{4}{*}{ TNF- $\alpha$} & \multirow{2}{*}{ EX } & PRE & $1.07 \pm 0.11$ & \multirow{2}{*}{1.000} \\
\hline & & POST & $1.05 \pm 0.10$ & \\
\hline & \multirow{2}{*}{ SED } & PRE & $1.37 \pm 0.18$ & \multirow{2}{*}{0.665} \\
\hline & & POST & $1.23 \pm 0.16$ & \\
\hline \multirow{4}{*}{ Caspase-1 } & \multirow{2}{*}{ EX } & PRE & $100.48 \pm 9.78$ & \multirow{2}{*}{0.217} \\
\hline & & POST & $112.97 \pm 9.81$ & \\
\hline & \multirow{2}{*}{ SED } & PRE & $121.61 \pm 25.43$ & \multirow{2}{*}{1.000} \\
\hline & & POST & $100.00 \pm 14.64$ & \\
\hline Molecule & Group & Time & Mean concentration $(\mathrm{ng} / \mathrm{mL}) \pm \mathrm{SEM}$ & $P$ values \\
\hline \multirow{4}{*}{ MMP-9 } & \multirow{2}{*}{ EX } & PRE & $192.35 \pm 23.43$ & 1.000 \\
\hline & & POST & $174.40 \pm 17.84$ & 1.000 \\
\hline & SED & PRE & $208.36 \pm 25.59$ & 0.368 \\
\hline & & POST & $176.32 \pm 20.86$ & - \\
\hline & EX & PRE & $16.11 \pm 1.65$ & 0.300 \\
\hline DHEA & & POST & $17.06 \pm 1.98$ & \\
\hline DHAT & SED & PRE & $16.08 \pm 2.17$ & 1000 \\
\hline & SDE & POST & $16.18 \pm 1.90$ & 1.000 \\
\hline Molecule & Group & Time & Mean concentration $(\mu \mathrm{g} / \mathrm{dL}) \pm \mathrm{SEM}$ & $P$ values \\
\hline & EX & PRE & $22.17 \pm 1.73$ & 0.047 \\
\hline Cortisol & & POST & $23.73 \pm 1.74$ & \\
\hline & SED & PRE & $20.07 \pm 1.00$ & 0.110 \\
\hline & & POST & $21.49 \pm 0.78$ & \\
\hline Molecule & Group & Time & Mean molar ratio \pm SEM & $P$ values \\
\hline & EX & PRE & $13.78 \pm 1.41$ & 0.716 \\
\hline Cortisol/DHEA & & POST & $14.50 \pm 1.57$ & \\
\hline & SED & PRE & $12.18 \pm 1.44$ & 0.234 \\
\hline & & POST & $12.80 \pm 1.34$ & \\
\hline
\end{tabular}

The serum concentration of signaling molecules was measured with ELISA, and the molar ratio of cortisol to DHEA was calculated. Results are shown as mean concentration or molar ratio \pm SEM.

IL, interleukin; TNF, tumour necrosis factor; MMP, matrix metalloproteinase; DHEA, dehydroepiandrosterone; EX, exercise group; SED, sedentary control group; PRE, baseline measurement; POST, measurement after 12 weeks; SEM, standard error of the mean.

concentration of IL-6, IL-10, IL-12p70, TNF- $\alpha$, caspase-1, and MMP-9 (Table 2).

3.3. Circulating $p D C$ Numbers Increase following Long-Term Physical Exercise in MS. We were not able to detect an effect of the physical exercise program on the absolute number of total leukocytes nor on the absolute number of lymphocytes, monocytes, and granulocytes (data not shown). Next, we investigated the number of circulating DC subsets, that is, $\mathrm{CDC}$ and $\mathrm{pDC}$, in MS patients participating in the combined exercise program (EX, $n=29)$ as compared to sedentary control patients (SED, $n=16$ ). Our findings demonstrate that, in the course of the 12-week time period, a difference between trained and sedentary patients 
could be demonstrated regarding the absolute number of $\mathrm{pDC}(P=0.017)$. Indeed, increased cell counts of $\mathrm{pDC}$ were detected in the exercise group $(P=0.010$; Figure $2(\mathrm{a}))$ after 12 weeks, while no changes were observed in the sedentary control group. Also the proportion of circulating $\mathrm{pDC}$ within total white blood cells was increased in MS patients who participated in the exercise program $(P=0.050$; data not shown). No effect of the 12 -week intervention on the absolute number of $\mathrm{cDC}$ could be detected. Both patient groups displayed an increase in the absolute number of cDC after 12 weeks (EX: $P=0.026$, SED: $P=0.002$; Figure 2(b)).

3.4. Increased Numbers of pDC with an Activated Phenotype Are Found following the Physical Exercise Program in MS. The absolute number of $\mathrm{CDC}$ and $\mathrm{pDC}$ positive for the costimulatory molecules CD80 and CD86 was analyzed by flow cytometry. In the course of the 12 -week time period, a difference was observed between the patient groups for the absolute number of $\mathrm{CD}^{+} 0^{+} \mathrm{pDC}(P=0.015)$ and of $\mathrm{CD} 86^{+}$ pDC $(P=0.016)$. Indeed, significantly increased numbers of $\mathrm{CD}^{+} 0^{+} \mathrm{pDC}$ were observed in MS patients following the 12week combined exercise program $(P<0.001$; Figure $2(\mathrm{c}))$, while no differences in the number of $\mathrm{CD}^{+} 0^{+} \mathrm{pDC}$ could be found in the sedentary control group. Similarly, also the proportion of circulating $\mathrm{CD}^{+} 0^{+} \mathrm{pDC}$ within total white blood cells was increased in MS patients who participated in the exercise program ( $P=0.030$; data not shown). Both intervention groups display, however, a decrease in $\mathrm{CD}^{+} 6^{+}$ pDC numbers after 12 weeks (EX: $P=0.046$, SED: $P=$ 0.001 ; Figure 2(c)), indicative of an effect that cannot be attributed to the physical exercise program. In addition, no differences in the course of the 12 -week time period could be detected between the patient groups for the absolute number of $\mathrm{CD}^{+} 0^{+}$and $\mathrm{CD} 86^{+} \mathrm{cDC}$, although $\mathrm{CD}^{+} 6^{+} \mathrm{cDC}$ significantly decreased after 12 weeks in the sedentary control group $(P=0.022$; Figure $2(\mathrm{~d}))$.

Simultaneously, the absolute number of $\mathrm{cDC}$ and $\mathrm{pDC}$ positive for the migration markers, CD62L, CCR5, and CCR7, was measured. No differences regarding the absolute number of $\mathrm{CD}_{2} \mathrm{~L}^{+}, \mathrm{CCR}^{+}$, and $\mathrm{CCR} 7^{+} \mathrm{pDC}$ were detected between the patient groups in the course of the 12-week time period. However, we did show a significantly increased number of $\mathrm{CD} 2 \mathrm{~L}^{+}$pDC in MS patients following the exercise program $(P=0.002$; Figure $2(\mathrm{e}))$, while no changes were observed in the sedentary control group. In addition, a modest but significant decrease in the number of $C C R 7^{+}$pDC could be detected after 12 weeks in the sedentary control group $(P=$ 0.009 ; Figure $2(\mathrm{e}))$. No differences regarding the absolute number of $\mathrm{CD} 2 \mathrm{~L}^{+}, \mathrm{CCR}^{+}$, and $\mathrm{CCR}^{+} \mathrm{cDC}$ could be detected between the groups in the course of the 12-week time period, although modest but significant changes in the number of $\mathrm{CD} 2 \mathrm{~L}^{+}$and $\mathrm{CCR} 5^{+} \mathrm{CDC}$ could be observed in the sedentary control group (CD62L: $P=0.041$, CCR5: $P=$ 0.025; Figure 2(f)).

Taken together, our observations show that increased numbers of $\mathrm{CD}^{+} 0^{+}$and $\mathrm{CD} 2 \mathrm{~L}^{+} \mathrm{pDC}$, indicative of an activated phenotype, are found following the exercise program.
3.5. TLR Responsiveness following Long-Term Physical Exercise in MS Is Altered. In order to assess the responsiveness of circulating DC to danger signals, blood samples were stimulated with a TLR4 ligand, LPS in combination with IFN$\gamma$, or a TLR7 ligand, IQ. Both ligands are known to activate $\mathrm{cDC}$ and pDC, respectively $[32,33]$. Following 12 weeks of exercise, the fold change of CCR $5^{+} \mathrm{CDC}$ upon LPS and IFN- $\gamma$ stimulation was decreased in the exercise group $(P=0.002$; Table 3 ) and significantly differed from the sedentary group in the course of the 12-week time period $(P=0.038)$, where no changes were observed. No differences regarding TNF$\alpha$ and MMP-9 secretion upon LPS and IFN- $\gamma$ stimulation could be detected between the patient groups in the course of the 12-week time period. Nevertheless, TNF- $\alpha$ and MMP-9 secretion was significantly decreased in trained MS patients (TNF- $\alpha$ : $P=0.028$; MMP-9: $P=0.040$ ), while no changes were detected in the sedentary control group (Table 4 ). In addition, no significant differences could be detected for the fold change of $\mathrm{CD}^{+} 6^{+}$and HLA-DR ${ }^{+} \mathrm{CDC}$, neither for the secreted amount of IL- $1 \beta$, IL-6, IL-12p70, IFN- $\alpha$, and caspase1 upon LPS and IFN- $\gamma$ stimulation between the trained and sedentary MS patients after 12 weeks (Tables 3 and 4).

In the course of the 12-week time period, the fold change of HLA-DR ${ }^{+}$pDC upon IQ stimulation differed significantly between the patient groups $(P=0.023)$. A significant increase in the fold change of HLA-DR ${ }^{+}$pDC upon IQ stimulation was observed in MS patients after 12 weeks of exercise $(P=0.008)$, but not in the sedentary control group (Table 3). Furthermore, IL-1 $\beta$ secretion upon IQ stimulation differed between the patient groups in the course of the 12week time period ( $P=0.039$; Table 4$)$. Nevertheless, after 12 weeks, IL-1 $\beta$ secretion was only significantly decreased in the sedentary control group $(P=0.029)$, indicative of an effect that cannot be attributed to the physical exercise program. No significant differences could be detected for the fold change of $\mathrm{CCR}^{+}$and $\mathrm{CD} 86^{+} \mathrm{pDC}$ nor for the secreted amount of IL6, IL-12p70, TNF- $\alpha$, IFN- $\alpha$, caspase-1, and MMP-9 upon IQ stimulation between trained and sedentary MS patients after 12 weeks (Tables 3 and 4 ).

3.6. Circulating Treg Subsets Are Not Affected by Long-Term Physical Exercise in MS. Using flow cytometry, we next characterized the effect of physical exercise on Treg subsets, that is, CD25 ${ }^{\text {hi }} \mathrm{FoxP}^{+}$, Tr1, and Th3 (Table 5).

In our hands, no effect of the 12-week combined exercise program on the proportion of Treg populations could be detected. Similarly, no effect was observed in the sedentary control group. Interestingly, a positive correlation was found at log-log scale between the proportion of IL-10-producing Tr1 cells and the number of $\mathrm{CD}^{+} 0^{+} \mathrm{pDC}(P=0.001, \rho=$ 0.383; Figure 3).

\section{Discussion}

Based on previously demonstrated improvements in muscle strength, cardiovascular health, mental functioning, and quality of life in MS patients participating in an exercise program, we examined the modulation of immune variables 
Plasmacytoid DC

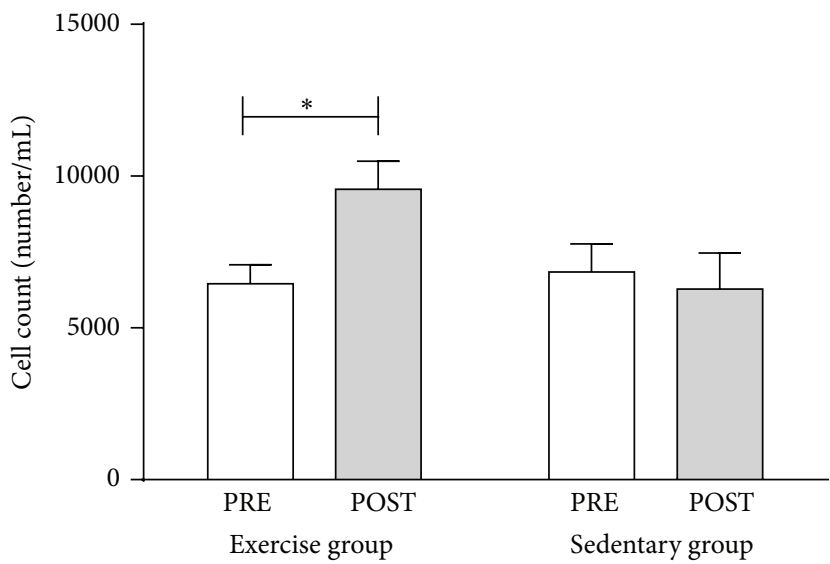

(a)

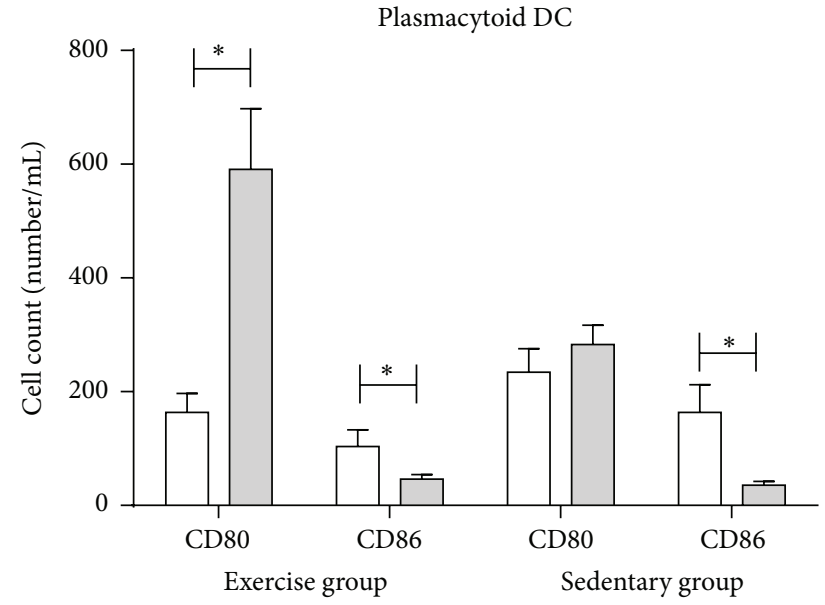

(c)

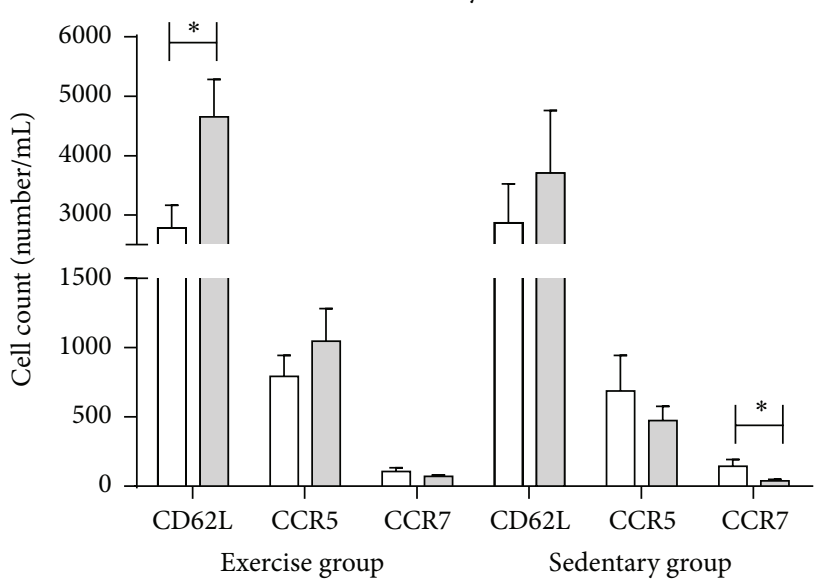

(e)

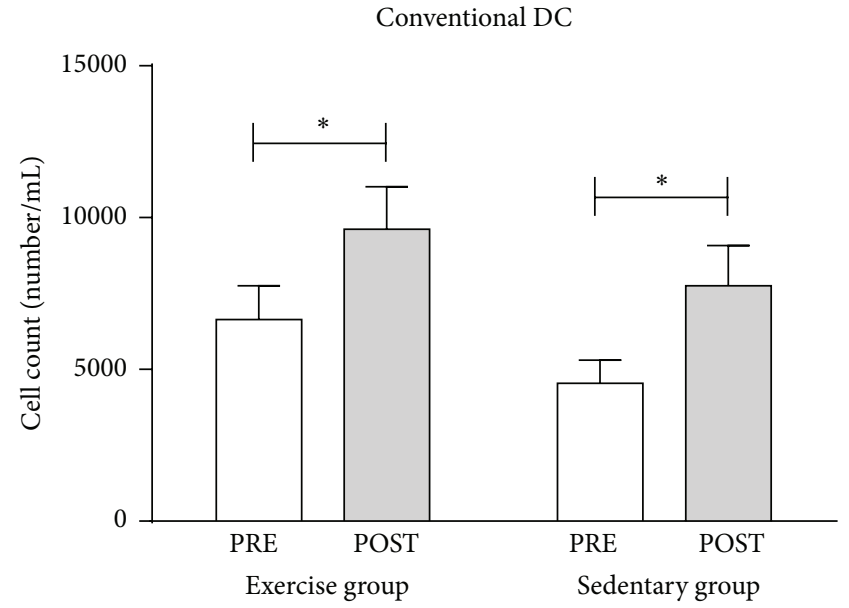

(b)

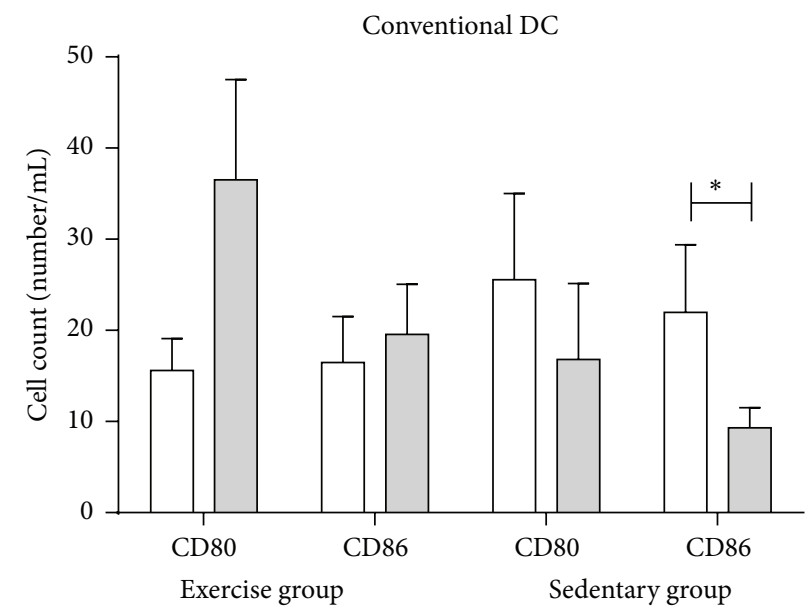

(d)

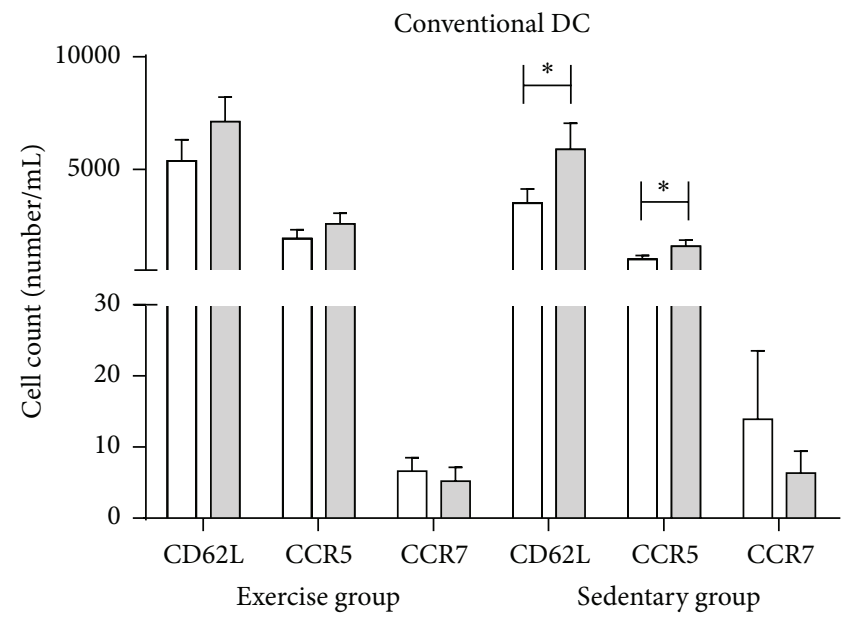

(f)

FIGURE 2: Increased numbers of activated pDC are found following a 12-week combined exercise program in MS. Enumeration of the absolute number of circulating pDC (a) and cDC (b) was determined by means of a double platform method using an automatic cell counter and flow cytometry before start of the study (open bars) and after 12 weeks (filled bars). The activation state (c, d) and the migratory profile (e, f) of DC were assessed by enumerating pDC and cDC expressing CD80, CD86, CD62L, CCR5, and CCR7. Results are shown as mean absolute number \pm SEM. ${ }^{*} P<0.05$. cDC, conventional dendritic cells; pDC, plasmacytoid dendritic cells; EX, exercise group; SED, sedentary control group; SEM, standard error of the mean. 
TABLE 3: A 12-week exercise program induces a decreased fold change of CCR5 ${ }^{+} \mathrm{cDC}$ upon LPS and IFN- $\gamma$ stimulation and an increased fold change of HLA-DR ${ }^{+}$pDC upon IQ stimulation.

\begin{tabular}{|c|c|c|c|c|c|c|}
\hline \multirow{2}{*}{ Marker } & \multirow{2}{*}{ Group } & \multirow{2}{*}{ Time } & \multicolumn{2}{|l|}{$\mathrm{cDC}$} & \multicolumn{2}{|l|}{$\mathrm{pDC}$} \\
\hline & & & Mean fold change \pm SEM & $P$ values & Mean fold change \pm SEM & $P$ values \\
\hline \multirow{4}{*}{ CCR5 } & \multirow{2}{*}{ EX } & PRE & $0.59 \pm 0.06$ & \multirow{2}{*}{0.002} & $1.45 \pm 0.28$ & \multirow{2}{*}{1.000} \\
\hline & & POST & $0.38 \pm 0.06$ & & $1.98 \pm 0.61$ & \\
\hline & \multirow{2}{*}{ SED } & PRE & $0.51 \pm 0.06$ & \multirow{2}{*}{1.000} & $1.67 \pm 0.37$ & \multirow{2}{*}{0.184} \\
\hline & & POST & $0.56 \pm 0.09$ & & $2.08 \pm 0.98$ & \\
\hline \multirow{4}{*}{ CD86 } & \multirow{2}{*}{ EX } & PRE & $2.68 \pm 0.45$ & \multirow{2}{*}{0.100} & $12.34 \pm 2.55$ & \multirow{2}{*}{0.383} \\
\hline & & POST & $2.12 \pm 0.23$ & & $9.14 \pm 2.11$ & \\
\hline & \multirow{2}{*}{ SED } & PRE & $2.44 \pm 0.48$ & \multirow{2}{*}{1.000} & $5.70 \pm 1.56$ & \multirow{2}{*}{0.767} \\
\hline & & POST & $2.84 \pm 0.69$ & & $5.95 \pm 2.73$ & \\
\hline \multirow{4}{*}{ HLA-DR } & \multirow{2}{*}{ EX } & PRE & $1.01 \pm 0.01$ & \multirow{2}{*}{0.189} & $0.90 \pm 0.03$ & \multirow{2}{*}{0.008} \\
\hline & & POST & $1.01 \pm 0.01$ & & $0.97 \pm 0.02$ & \\
\hline & \multirow{2}{*}{ SED } & PRE & $1.06 \pm 0.06$ & \multirow{2}{*}{0.439} & $0.92 \pm 0.06$ & \multirow{2}{*}{0.609} \\
\hline & & POST & $1.06 \pm 0.03$ & & $0.82 \pm 0.08$ & \\
\hline
\end{tabular}

Blood samples were stimulated overnight with LPS in combination with IFN- $\gamma$, or IQ. The percentage of CCR5, CD86, and HLA-DR positive cDC and pDC after TLR stimulation was measured using flow cytometry. Mean fold changes are calculated as the ratio of the proportion of CCR5, CD86, and HLA-DR positive $\mathrm{CDC}$ and $\mathrm{pDC}$ in the stimulated condition to the nonstimulated condition. Results are shown as mean fold change \pm SEM.

cDC, conventional dendritic cells; pDC, plasmacytoid dendritic cells; LPS, lipopolysaccharide; IQ, imiquimod; EX, exercise group; SED, sedentary group; PRE, baseline measurement; POST, measurement after 12 weeks; SEM, standard error of the mean.

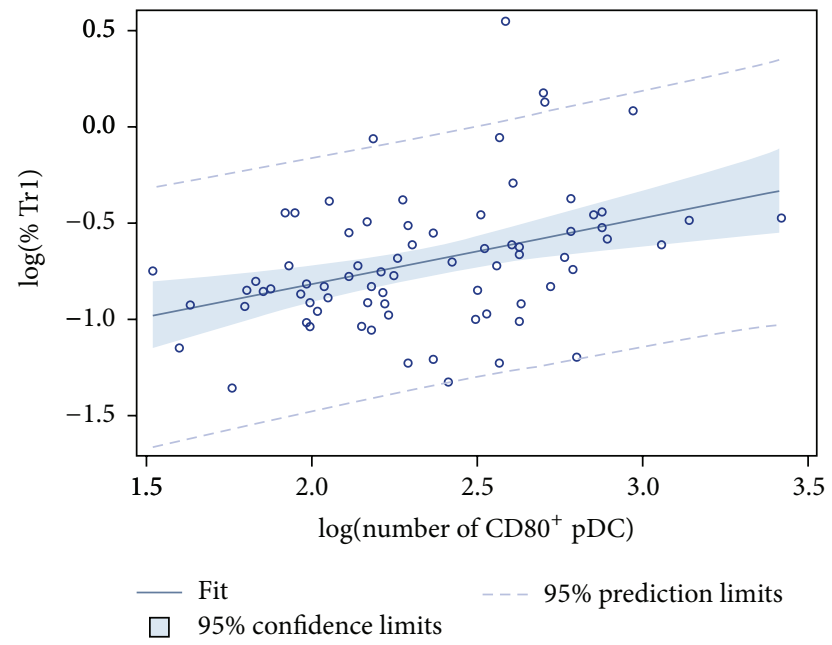

Figure 3: Positive correlation at log-log scale between the proportion of $\operatorname{Trl}$ and the number of $\mathrm{CD}^{+} 0^{+}$pDC. A positive correlation at log-log scale was found between the proportion of $\operatorname{Trl}$ cells and the number of $\mathrm{CD}^{+} 0^{+} \mathrm{pDC}(P=0.001 ; \rho=0.383)$. Both variables were log-transformed to obtain a normal distribution. pDC, plasmacytoid dendritic cells; Tr1, type 1 regulatory T cell; log, logarithmic transformation; $\rho$, Pearson's correlation coefficient.

that are known to impact disease activity in MS, namely, cellular and molecular mediators of innate immunity, following a 12-week combined exercise program in this study. This hypothesis is supported by studies investigating the effect of physical exercise in the experimental autoimmune encephalomyelitis (EAE) animal model for MS [8].

We were not able to detect differences following 12 weeks of exercise for the IL- 6 and IL-12p70 serum concentration, similar to findings made by others [34-36]. Likewise, no differences in the serum concentration of IL-10, TNF- $\alpha$, caspase-1, and MMP-9 were found following the exercise program. After an exercise program in MS patients, White et al. found decreased IL-10 serum levels [35], while TNF$\alpha$ serum levels increased [36], decreased [35], or remained unchanged [34]. MMP-9 serum levels increased [37] or decreased [38] following an exercise program in healthy men and men with metabolic syndrome, respectively. The different outcomes of the various studies could be attributed to the variability in training protocols and study populations or to the different techniques used to quantify the secreted levels of inflammatory mediators.

Interestingly, we demonstrated decreased TNF- $\alpha$ and MMP-9 secretion following whole blood stimulation with LPS and IFN- $\gamma$ in trained MS patients, which may be of potential interest in disease-mediated pathways. We hypothesize that the observed reduced secretion of TNF- $\alpha$ might contribute to a diminished stimulation of myelin-specific Th1 and Th17 effector cells, ultimately resulting in attenuated disease activity. In support of this, reduced frequencies of LPS- and IFN- $\gamma$-stimulated cytokine-producing monocytes and $\mathrm{CDC}$ were found in elite swimmers after long-term intensive training [39]. Furthermore, reduced LPS-induced cytokine production by monocyte-derived DC resulted in diminished stimulation of Th1 and Th17 effector cells [40]. In addition, the observed reduced secretion of MMP-9 might positively affect blood-brain barrier integrity. Indeed, the primary role of MMP-9 is degradation of the extracellular matrix, for example, during blood-brain barrier transmigration of leukocytes to the CNS, which is seen in MS [41]. Together with the significantly more pronounced reduction in $\mathrm{CCR}^{+} \mathrm{cDC}$ upon LPS and IFN- $\gamma$ stimulation observed in MS patients who participated in the exercise program, this may suggest that exercise affects the migratory potential of cDC, although functional studies are needed to confirm 
TABLE 4: Exercise results in decreased secretion of the inflammatory mediators, TNF- $\alpha$ and MMP-9, upon LPS and IFN- $\gamma$ stimulation.

\begin{tabular}{|c|c|c|c|c|c|c|}
\hline \multirow{2}{*}{ Inflammatory mediator } & \multirow{2}{*}{ Group } & \multirow{2}{*}{ Time } & \multicolumn{2}{|l|}{ LPS + IFN- $\gamma$} & \multicolumn{2}{|l|}{ IQ } \\
\hline & & & Mean fold change \pm SEM & $P$ values & Mean fold change \pm SEM & $P$ values \\
\hline \multirow{4}{*}{ IL-1 $\beta$} & \multirow{2}{*}{ EX } & PRE & $89.3 \pm 11.5$ & \multirow{2}{*}{0.461} & $2.2 \pm 0.2$ & \multirow{2}{*}{0.790} \\
\hline & & POST & $77.1 \pm 11.5$ & & $2.0 \pm 0.2$ & \\
\hline & \multirow{2}{*}{ SED } & PRE & $94.1 \pm 19.5$ & \multirow{2}{*}{0.258} & $2.7 \pm 0.6$ & \multirow{2}{*}{0.029} \\
\hline & & POST & $72.0 \pm 16.9$ & & $1.8 \pm 0.4$ & \\
\hline \multirow{4}{*}{ IL-6 } & \multirow{2}{*}{ EX } & PRE & $111.0 \pm 7.7$ & \multirow{2}{*}{1.000} & $15.7 \pm 2.1$ & \multirow{2}{*}{1.000} \\
\hline & & POST & $110.8 \pm 6.5$ & & $15.9 \pm 2.5$ & \\
\hline & \multirow{2}{*}{ SED } & PRE & $103.5 \pm 12.8$ & \multirow{2}{*}{0.782} & $16.8 \pm 3.1$ & \multirow{2}{*}{1.000} \\
\hline & & POST & $109.7 \pm 11.0$ & & $14.5 \pm 3.4$ & \\
\hline \multirow{4}{*}{ IL-12p70 } & \multirow{2}{*}{ EX } & PRE & $26.0 \pm 7.6$ & \multirow{2}{*}{0.313} & $1.2 \pm 0.1$ & \multirow{2}{*}{1.000} \\
\hline & & POST & $15.2 \pm 4.1$ & & $1.2 \pm 0.2$ & \\
\hline & \multirow{2}{*}{ SED } & PRE & $18.1 \pm 6.4$ & \multirow{2}{*}{1.000} & $1.6 \pm 0.4$ & \multirow{2}{*}{0.410} \\
\hline & & POST & $19.5 \pm 6.8$ & & $2.2 \pm 0.6$ & \\
\hline \multirow{4}{*}{ TNF- $\alpha$} & \multirow{2}{*}{ EX } & PRE & $55.1 \pm 5.7$ & \multirow{2}{*}{0.028} & $1.6 \pm 0.1$ & \multirow{2}{*}{0.926} \\
\hline & & POST & $39.2 \pm 4.4$ & & $1.5 \pm 0.1$ & \\
\hline & \multirow{2}{*}{ SED } & PRE & $43.9 \pm 8.0$ & 1000 & $1.6 \pm 0.2$ & 0.168 \\
\hline & & POST & $46.5 \pm 9.3$ & 1.000 & $1.3 \pm 0.2$ & \\
\hline & EX & PRE & $2.1 \pm 0.6$ & 1000 & $7.4 \pm 1.2$ & 1000 \\
\hline JFN_ $\alpha$ & $\mathrm{E} \Lambda$ & POST & $2.7 \pm 0.9$ & 1.000 & $8.0 \pm 1.6$ & 1.000 \\
\hline $11+20$ & SED & PRE & $1.9 \pm 0.8$ & 1.000 & $6.7 \pm 1.8$ & 0.231 \\
\hline & & POST & $1.7 \pm 0.6$ & & $4.4 \pm 1.0$ & \\
\hline & $\mathrm{EX}$ & PRE & $8.8 \pm 0.7$ & 0.604 & $4.8 \pm 0.7$ & 0.260 \\
\hline Caspase-1 & & POST & $8.0 \pm 0.7$ & & $3.6 \pm 0.4$ & \\
\hline & SED & PRE & $8.1 \pm 1.0$ & 1.000 & $3.6 \pm 0.6$ & 1.000 \\
\hline & & POST & $6.8 \pm 0.8$ & & $3.8 \pm 0.9$ & \\
\hline & EX & PRE & $7.9 \pm 0.8$ & 0.040 & $2.2 \pm 0.2$ & 1.000 \\
\hline MMP_9 & & POST & $6.2 \pm 0.5$ & 0.040 & $2.1 \pm 0.1$ & 1.000 \\
\hline & SED & PRE & $6.5 \pm 1.0$ & 0.233 & $2.0 \pm 0.2$ & 1.000 \\
\hline & & POST & $6.4 \pm 0.6$ & & $2.0 \pm 0.3$ & \\
\hline
\end{tabular}

Blood samples were stimulated overnight with LPS in combination with IFN- $\gamma$, or IQ. Secretion of inflammatory mediators was quantified using ELISA. Mean fold changes are calculated as the ratio of the secreted concentration in the stimulated condition to the nonstimulated condition. Results are shown as mean fold change \pm SEM.

LPS, lipopolysaccharide; IQ, imiquimod; IL, interleukin; TNF, tumor necrosis factor; IFN, interferon; MMP, matrix metalloproteinase; EX, exercise group; SED, sedentary control group; PRE, baseline measurement; POST, measurement after 12 weeks; SEM, standard error of the mean.

TABLE 5: Circulating Treg subsets are not affected by long-term physical exercise in MS.

\begin{tabular}{|c|c|c|c|c|}
\hline Treg subset & Group & Time & Mean $\%$ Treg in $\mathrm{CD}^{+} \mathrm{T}$ cell population $\pm \mathrm{SEM}$ & $P$ values \\
\hline \multirow{4}{*}{$\mathrm{CD}_{2} 5^{\mathrm{hi}} \mathrm{FoxP}^{+}$} & \multirow{2}{*}{ EX } & PRE & $0.62 \pm 0.11$ & \multirow{2}{*}{0.083} \\
\hline & & POST & $1.12 \pm 0.41$ & \\
\hline & \multirow{2}{*}{ SED } & PRE & $1.00 \pm 0.30$ & \multirow{2}{*}{0.782} \\
\hline & & POST & $1.69 \pm 0.85$ & \\
\hline \multirow{4}{*}{ IL-10 $0^{+}(\operatorname{Tr} 1)$} & \multirow{2}{*}{ EX } & PRE & $0.22 \pm 0.05$ & \multirow{2}{*}{1.000} \\
\hline & & POST & $0.20 \pm 0.02$ & \\
\hline & \multirow{2}{*}{ SED } & PRE & $0.33 \pm 0.09$ & \multirow{2}{*}{0.796} \\
\hline & & POST & $0.50 \pm 0.26$ & \\
\hline \multirow{4}{*}{ TGF- $\beta^{+}$(Th3) } & FX & PRE & $0.15 \pm 0.02$ & \multirow{2}{*}{1.000} \\
\hline & & POST & $0.19 \pm 0.09$ & \\
\hline & \multirow{2}{*}{ SED } & PRE & $0.17 \pm 0.04$ & \multirow{2}{*}{0.056} \\
\hline & & POST & $0.12 \pm 0.08$ & \\
\hline
\end{tabular}

The percentage of circulating Treg subsets was determined using flow cytometry. Results are shown as mean percentage \pm SEM.

Treg, regulatory T cell; FoxP3, forkhead box P3; IL, interleukin; Trl, type 1 regulatory T cell; TGF, transforming growth factor; Th3, T helper 3 cell; EX, exercise group; SED, sedentary control group; PRE, baseline measurement; POST, measurement after 12 weeks; SEM, standard error of the mean. 
this. In contrast to our findings, a reduction in LPS-induced secretion of IL- $1 \beta$, IL-6, and IL-12 was observed after longterm physical exercise by others [39]. Nevertheless, further experiments are mandatory to identify the responsible cell types secreting the inflammatory mediators as well as to determine whether long-term exercise affects the surface expression of TLR resulting in the observed reduced secretion of inflammatory mediators. Indeed, whereas higher expression levels of TLR2 and TLR4 were found on mononuclear cells in MS patients as compared to healthy controls [42], a reduction in the expression levels of TLR could potentially mediate the favourable effect of physical exercise in MS patients.

No effect of exercise on the absolute number of $\mathrm{cDC}$ could be detected, as indicated by an increase in $\mathrm{CDC}$ numbers after the 12 -week time period in both study groups. It has been demonstrated that changes in frequency of circulating myeloid cells correlate with certain chronic diseases [43]. Previously, we showed decreased numbers of circulating pDC in MS patients as compared to healthy controls [22]. Interestingly, in this study, increased cell counts of pDC, more specifically $\mathrm{CD}^{+} 0^{+} \mathrm{pDC}$ and $\mathrm{CD}^{2} 2 \mathrm{~L}^{+} \mathrm{pDC}$, were found after 12 weeks of exercise, indicative of an activated phenotype. However, others have reported conflicting results. Indeed, Chiang et al. found that healthy practitioners of Tai-Chi Chuan, an aerobic exercise of moderate intensity, showed increased numbers of circulating $\mathrm{CDC}$, but not $\mathrm{pDC}$, as compared to sedentary individuals [26]. Also in obese individuals an increased proportion of $\mathrm{CDC}$, but a decreased proportion of $\mathrm{pDC}$, was observed after 10 weeks of intensified exercise training [27]. Noteworthy, in the same study no differences regarding $\mathrm{CDC}$ and $\mathrm{pDC}$ numbers were observed in lean individuals. However, a sedentary control group was not included [27]. Others recently demonstrated that natalizumab treatment of MS also increases the number of circulating pDC [44]. However, in our hands no effect of natalizumab treatment on the reported findings could be observed (data not shown). Furthermore, we observed a significant increase in the fold change of $\mathrm{HLA}-\mathrm{DR}^{+} \mathrm{pDC}$ upon IQ stimulation after completion of the exercise program. This may suggest an increase in the number of $\mathrm{pDC}$ contributing to antigen presentation upon TLR stimulation in MS patients who participated in the exercise program.

Based on our observations demonstrating increased numbers of activated pDC following the 12-week combined exercise program as well as on the role of activated $\mathrm{pDC}$ in inducing IL-10-producing Treg (Tr1) [21], we hypothesized increased numbers of Tr1 in MS patients who completed the exercise program. Whereas in our hands no effect of long-term physical exercise on the proportion of Treg populations could be observed, others previously reported that individuals who participated in a long-term physical exercise program show higher proportions of circulating Treg [45]. Importantly, we demonstrate a positive correlation between the proportion of $\operatorname{Tr} 1$ and the number of circulating $\mathrm{CD}^{+} 0^{+}$ pDC, underscoring the hypothesis that increasing numbers of activated pDC may drive the induction of Treg following long-term physical exercise in MS. However, the role of $\mathrm{pDC}$ in immunity and tolerance appears complex and activated
pDC also participate in innate immunity with a specialized production of type I IFN. In doing so, pDC can stimulate the proliferation of naive $\mathrm{T}$ cells [46], albeit poorly relative to cDC. Nevertheless, no effect on the secretion of IFN$\alpha$ by pDC upon TLR7 stimulation was observed following physical exercise in this study. Hence, it is less likely that increased activated pDC numbers induce effector $\mathrm{T}$ cell stimulation following long-term physical exercise, further supporting promotion of the immunoregulatory function of pDC. Future experiments are needed to understand the role of $\mathrm{pDC}$ in managing both innate and adaptive immune responses, for example, expression of inducible tolerogenic enzyme indoleamine 2,3-dioxygenase (IDO), inducible costimulator ligand (ICOS-L), and/or programmed death 1 ligand (PD-L1), which mediate Treg development, and how this is affected by long-term exercise. Additional prognostic studies addressing associations between immunologic effects of long-term exercise and clinical changes in MS patients are needed.

In this respect, it has been proposed that exercise stress influences the immune system via the activation of the HPA axis and the sympathetic nervous system, two main neuroendocrine pathways [47]. It is known that their respective signaling molecules, cortisol, DHEA, and catecholamines modulate the number, functioning, trafficking, and activity of immune cells. Hence, evaluating the hormonal release could offer insight into the mechanisms that underlie exercisemediated immune modulation. Unfortunately, in our hands, no physiologically relevant changes in serum cortisol and DHEA levels after the exercise program could be observed, in line with other studies [48]. However, it is possible that a change in serum cortisol and DHEA levels is only elicited by a more intensive training program, as demonstrated by others for cortisol [39]. Finally, future studies should test different exercise intensities, to define a minimal exercise intensity where effects on the immune and/or neuroendocrine system, for example, cortisol, DHEA, and catecholamines, can be detected.

In conclusion, we confirmed that long-term physical exercise is safe in MS patients [49], as evidenced by no changes observed in the serum levels of proinflammatory mediators following the exercise program. In addition, the annualized relapse rate was not significantly different between the exercise ( 0.45 per year) and the sedentary group (0.35 per year). However, long-term follow-up of patients participating in physical exercise programs remains necessary. Based on our observations, we conclude a shift towards a tolerogenic profile after physical exercise, suggesting a favourable immunologic effect in MS. Indeed, we demonstrated increased numbers of activated pDC, associated with Tr1 development, and decreased secretion of TLR-induced inflammatory mediators in MS patients following the 12-week combined endurance and resistance exercise program. Noteworthy, whether these immune-modulatory effects occur in every subject undergoing a training program or are specific for MS patients needs to be addressed in future studies. Overall, our results suggest that long-term physical exercise favourably impacts the underlying immunopathogenesis of MS. 


\section{Conflict of Interests}

The authors declare that there is no conflict of interests regarding the publication of this paper.

\section{Acknowledgments}

The authors would like to thank all MS patients for participating in this study. This work was supported by Grant no. G.0168.09 of the Fund for Scientific Research-Flanders, Belgium (FWO-Vlaanderen). Further support was provided through the Special Research Fund (BOF), a BOF-GOA grant (ID PS 28313), Medical Legacy Fund (UZA), the Methusalem Funding Program, the Belgian Hercules Foundation, the Belgian Charcot Foundation, and the "Belgische Stichting Roeping." Amber H. Nuyts held a Ph.D. fellowship from the Flemish Institute for Science and Technology (IWT). Nathalie Cools held a postdoctoral fellowship of the Fund for Scientific Research-Flanders (FWO-Vlaanderen).

\section{References}

[1] A. Compston and A. Coles, "Multiple sclerosis," The Lancet, vol. 359, no. 9313, pp. 1221-1231, 2002.

[2] U. Dalgas, E. Stenager, and T. Ingemann-Hansen, "Multiple sclerosis and physical exercise: recommendations for the application of resistance-, endurance- and combined training," Multiple Sclerosis, vol. 14, no. 1, pp. 35-53, 2008.

[3] T. Broekmans, M. Roelants, P. Feys et al., "Effects of long-term resistance training and simultaneous electro-stimulation on muscle strength and functional mobility in multiple sclerosis," Multiple Sclerosis, vol. 17, no. 4, pp. 468-477, 2011.

[4] B. D. Çakt, B. Nacir, H. Genç et al., "Cycling progressive resistance training for people with multiple sclerosis: a randomized controlled study," American Journal of Physical Medicine and Rehabilitation, vol. 89, no. 6, pp. 446-457, 2010.

[5] N. M. Stroud and C. L. Minahan, “The impact of regular physical activity on fatigue, depression and quality of life in persons with multiple sclerosis," Health and Quality of Life Outcomes, vol. 7, article 68, 2009.

[6] I. Wens, D. Hansen, K. Verboven et al., "Impact of 24 weeks of resistance and endurance exercise on glucose tolerance in persons with multiple sclerosis," American Journal of Physical Medicine \& Rehabilitation, vol. 94, no. 10, supplement 1, pp. 838847, 2015.

[7] D. J. P. Garner and J. J. Widrick, "Cross-bridge mechanisms of muscle weakness in multiple sclerosis," Muscle and Nerve, vol. 27, no. 4, pp. 456-464, 2003.

[8] W. M. Pryor, K. G. Freeman, R. D. Larson, G. L. Edwards, and L. J. White, "Chronic exercise confers neuroprotection in experimental autoimmune encephalomyelitis," Journal of Neuroscience Research, vol. 93, no. 5, pp. 697-706, 2015.

[9] G. Giovannoni and G. Ebers, "Multiple sclerosis: the environment and causation," Current Opinion in Neurology, vol. 20, no. 3, pp. 261-268, 2007.

[10] D. Giunti, G. Borsellino, R. Benelli et al., "Phenotypic and functional analysis of T cells homing into the CSF of subjects with inflammatory diseases of the CNS," Journal of Leukocyte Biology, vol. 73, no. 5, pp. 584-590, 2003.
[11] R. Gandhi, A. Laroni, and H. L. Weiner, "Role of the innate immune system in the pathogenesis of multiple sclerosis," Journal of Neuroimmunology, vol. 221, no. 1-2, pp. 7-14, 2010.

[12] N. Cools, A. Petrizzo, E. Smits et al., "Dendritic cells in the pathogenesis and treatment of human diseases: a Janus Bifrons?" Immunotherapy, vol. 3, no. 10, pp. 1203-1222, 2011.

[13] K. P. A. MacDonald, D. J. Munster, G. J. Clark, A. Dzionek, J. Schmitz, and D. N. J. Hart, "Characterization of human blood dendritic cell subsets," Blood, vol. 100, no. 13, pp. 4512-4520, 2002.

[14] P. Hollender, D. Ittelet, F. Villard, J.-C. Eymard, P. Jeannesson, and J. Bernard, "Active matrix metalloprotease-9 in and migration pattern of dendritic cells matured in clinical grade culture conditions," Immunobiology, vol. 206, no. 4, pp. 441-458, 2002.

[15] M. Cella, D. Jarrossay, F. Faccheth et al., "Plasmacytoid monocytes migrate to inflamed lymph nodes and produce large amounts of type I interferon," Nature Medicine, vol. 5, no. 8, pp. 919-923, 1999.

[16] K. B. Elkon and A. Wiedeman, "Type I IFN system in the development and manifestations of SLE," Current Opinion in Rheumatology, vol. 24, no. 5, pp. 499-505, 2012.

[17] N. M. Rogers, J. S. Isenberg, and A. W. Thomson, "Plasmacytoid dendritic cells: no longer an enigma and now key to transplant tolerance?" American Journal of Transplantation, vol. 13, no. 5, pp. 1125-1133, 2013.

[18] J. J. Karrich, L. C. M. Jachimowski, C. H. Uittenbogaart, and B. Blom, "The plasmacytoid dendritic cell as the swiss army knife of the immune system: molecular regulation of its multifaceted functions," Journal of Immunology, vol. 193, no. 12, pp. 57725778, 2014.

[19] A. Goubier, B. Dubois, H. Gheit et al., "Plasmacytoid dendritic cells mediate oral tolerance," Immunity, vol. 29, no. 3, pp. 464475, 2008.

[20] B. M. Matta, A. Castellaneta, and A. W. Thomson, “Tolerogenic plasmacytoid DC," European Journal of Immunology, vol. 40, no. 10, pp. 2667-2676, 2010.

[21] T. Ito, M. Yang, Y.-H. Wang et al., "Plasmacytoid dendritic cells prime IL-10-producing T regulatory cells by inducible costimulator ligand," Journal of Experimental Medicine, vol. 204, no. 1, pp. 105-115, 2007.

[22] K. Thewissen, A. H. Nuyts, N. Deckx et al., "Circulating dendritic cells of multiple sclerosis patients are proinflammatory and their frequency is correlated with MS-associated genetic risk factors," Multiple Sclerosis, vol. 20, no. 5, pp. 548-557, 2014.

[23] M. Stasiolek, A. Bayas, N. Kruse et al., "Impaired maturation and altered regulatory function of plasmacytoid dendritic cells in multiple sclerosis," Brain, vol. 129, no. 5, pp. 1293-1305, 2006.

[24] N. P. Walsh, M. Gleeson, R. J. Shephard et al., "Position statement. Part one. Immune function and exercise," Exercise Immunology Review, vol. 17, pp. 6-63, 2011.

[25] L.-M. Chiang, Y.-J. Chen, J. Chiang, L.-Y. Lai, Y.-Y. Chen, and H.-F. Liao, "Modulation of dendritic cells by endurance training," International Journal of Sports Medicine, vol. 28, no. 9, pp. 798-803, 2007.

[26] J. Chiang, Y.-Y. Chen, T. Akiko et al., "Tai Chi Chuan increases circulating myeloid dendritic cells," Immunological Investigations, vol. 39, no. 8, pp. 863-873, 2010.

[27] T. Nickel, H. Hanssen, I. Emslander et al., "Immunomodulatory effects of aerobic training in obesity," Mediators of Inflammation, vol. 2011, Article ID 308965, 10 pages, 2011. 
[28] C. H. Polman, S. C. Reingold, B. Banwell et al., "Diagnostic criteria for multiple sclerosis: 2010 revisions to the McDonald criteria," Annals of Neurology, vol. 69, no. 2, pp. 292-302, 2011.

[29] M. E. Bauer, "Chronic stress and immunosenescence: a review," Neuroimmunomodulation, vol. 15, no. 4-6, pp. 241-250, 2008.

[30] C. M. Moriguchi Jeckel, R. P. Lopes, M. C. Berleze et al., "Neuroendocrine and immunological correlates of chronic stress in 'strictly healthy' populations,' NeuroImmunoModulation, vol. 17, no. 1, pp. 9-18, 2010.

[31] G. Verbeke and G. Molenberghs, Linear Mixed Models for Longitudinal Data, Springer, New York, NY, USA, 2000.

[32] J. Banchereau, F. Briere, C. Caux et al., "Immunobiology of dendritic cells," Annual Review of Immunology, vol. 18, pp. 767811,2000 .

[33] D. F. Tough, "Type I interferon as a link between innate and adaptive immunity through dendritic cell stimulation," Leukemia and Lymphoma, vol. 45, no. 2, pp. 257-264, 2004.

[34] J. Bansi, W. Bloch, U. Gamper, and J. Kesselring, “Training in MS: influence of two different endurance training protocols (aquatic versus overland) on cytokine and neurotrophin concentrations during three week randomized controlled trial," Multiple Sclerosis, vol. 19, no. 5, pp. 613-621, 2013.

[35] L. J. White, V. Castellano, and S. C. McCoy, "Cytokine responses to resistance training in people with multiple sclerosis," Journal of Sports Sciences, vol. 24, no. 8, pp. 911-914, 2006.

[36] V. Castellano, D. I. Patel, and L. J. White, "Cytokine responses to acute and chronic exercise in multiple sclerosis," Journal of Applied Physiology, vol. 104, no. 6, pp. 1697-1702, 2008.

[37] M. L. Urso, J. R. Pierce, J. A. Alemany, E. A. Harman, and B. C. Nindl, "Effects of exercise training on the matrix metalloprotease response to acute exercise," European Journal of Applied Physiology, vol. 106, no. 5, pp. 655-663, 2009.

[38] C. K. Roberts, D. Won, S. Pruthi et al., "Effect of a short-term diet and exercise intervention on oxidative stress, inflammation, MMP-9, and monocyte chemotactic activity in men with metabolic syndrome factors," Journal of Applied Physiology, vol. 100, no. 5, pp. 1657-1665, 2006.

[39] J. M. Morgado, L. Rama, I. Silva et al., "Cytokine production by monocytes, neutrophils, and dendritic cells is hampered by long-term intensive training in elite swimmers," European Journal of Applied Physiology, vol. 112, no. 2, pp. 471-482, 2012.

[40] E. Draper, J. DeCourcey, S. C. Higgins et al., "Conjugated linoleic acid suppresses dendritic cell activation and subsequent Th17 responses," Journal of Nutritional Biochemistry, vol. 25, no. 7, pp. 741-749, 2014.

[41] J.-H. Yen, T. Khayrullina, and D. Ganea, "PGE2-induced metalloproteinase-9 is essential for dendritic cell migration," Blood, vol. 111, no. 1, pp. 260-270, 2008.

[42] S. J. Hasheminia, S. H. Zarkesh-Esfahani, S. Tolouei, V. Shaygannejad, H. Shirzad, and C. M. Hashemzadeh, "Toll like receptor 2 and 4 expression in peripheral blood mononuclear cells of multiple sclerosis patients," Iranian Journal of Immunology, vol. 11, pp. 74-83, 2014.

[43] C. P. Verschoor, J. Johnstone, J. Millar et al., "Alterations to the frequency and function of peripheral blood monocytes and associations with chronic disease in the advanced-age, frail elderly," PLoS ONE, vol. 9, no. 8, Article ID e104522, 2014.

[44] P. Kivisäkk, K. Francois, J. Mbianda, R. Gandhi, H. L. Weiner, and S. J. Khoury, "Effect of natalizumab treatment on circulating plasmacytoid dendritic cells: a cross-sectional observational study in patients with multiple sclerosis," PLoS ONE, vol. 9, no. 7, Article ID e103716, 2014.
[45] S.-H. Yeh, H. Chuang, L.-W. Lin, C.-Y. Hsiao, P.-W. Wang, and K. D. Yang, "Tai chi chuan exercise decreases A1C levels along with increase of regulatory T-cells and decrease of cytotoxic Tcell population in type 2 diabetic patients," Diabetes Care, vol. 30, no. 3, pp. 716-718, 2007.

[46] J. A. Villadangos and L. Young, "Antigen-presentation properties of plasmacytoid dendritic cells," Immunity, vol. 29, no. 3, pp. 352-361, 2008.

[47] N. Deckx, W.-P. Lee, Z. N. Berneman, and N. Cools, "Neuroendocrine immunoregulation in multiple sclerosis," Clinical and Developmental Immunology, vol. 2013, Article ID 705232, 23 pages, 2013.

[48] J. L. J. Heaney, D. Carroll, and A. C. Phillips, "DHEA, DHEA$S$ and cortisol responses to acute exercise in older adults in relation to exercise training status and sex," Age, vol. 35, no. 2, pp. 395-405, 2013.

[49] L. A. Pilutti, M. E. Platta, R. W. Motl, and A. E. LatimerCheung, "The safety of exercise training in multiple sclerosis: a systematic review," Journal of the Neurological Sciences, vol. 343, no. 1-2, pp. 3-7, 2014. 


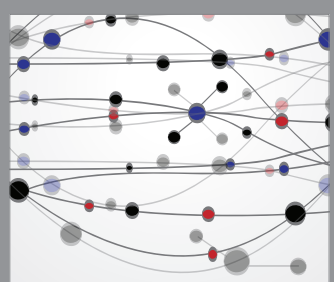

The Scientific World Journal
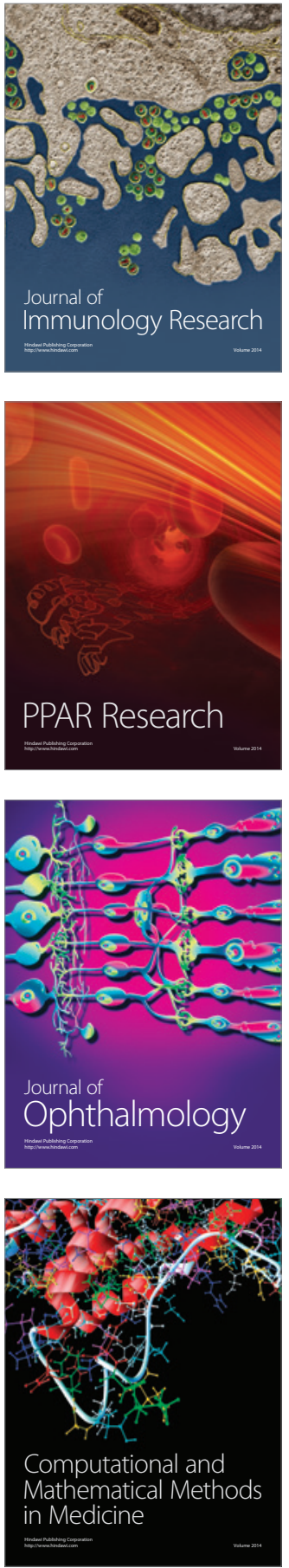

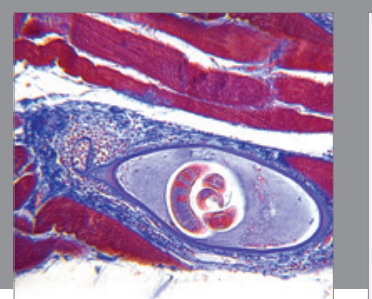

Gastroenterology Research and Practice

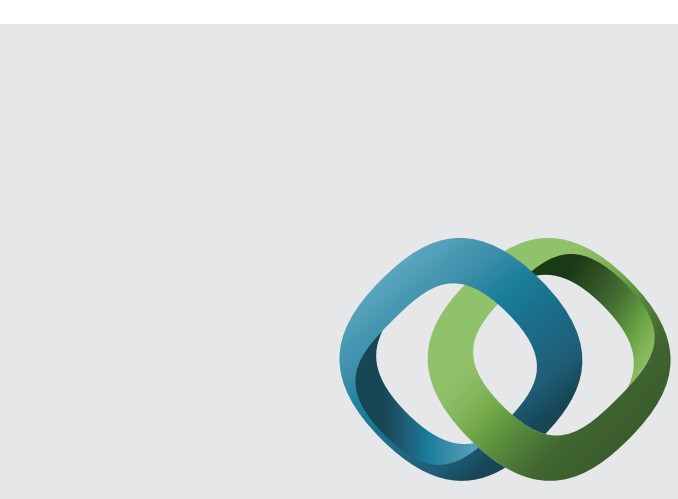

\section{Hindawi}

Submit your manuscripts at

http://www.hindawi.com
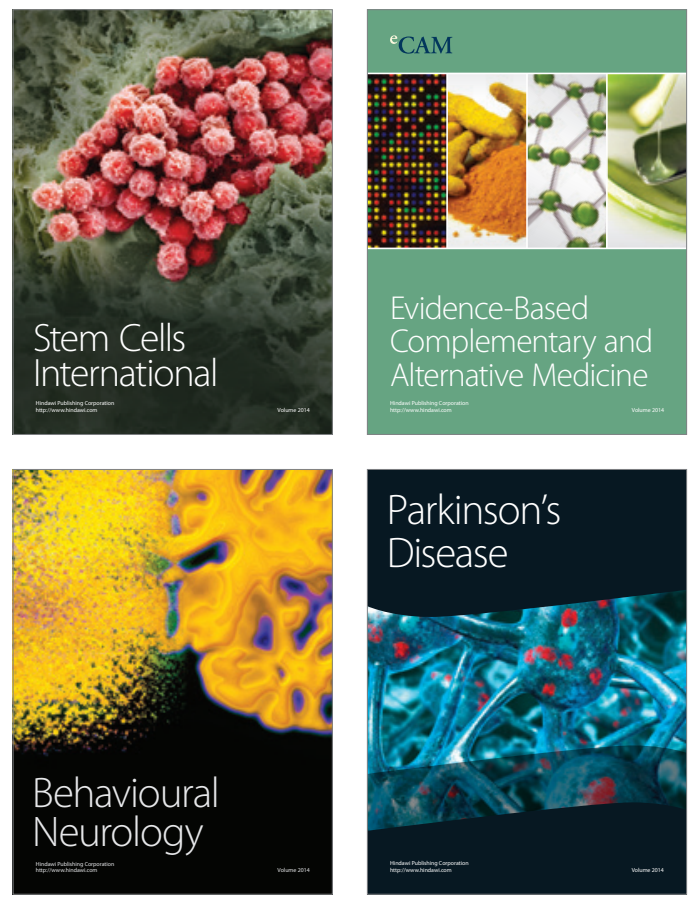
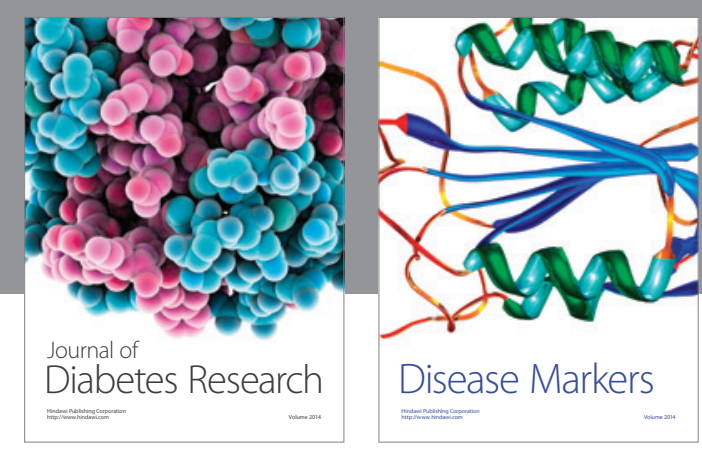

Disease Markers
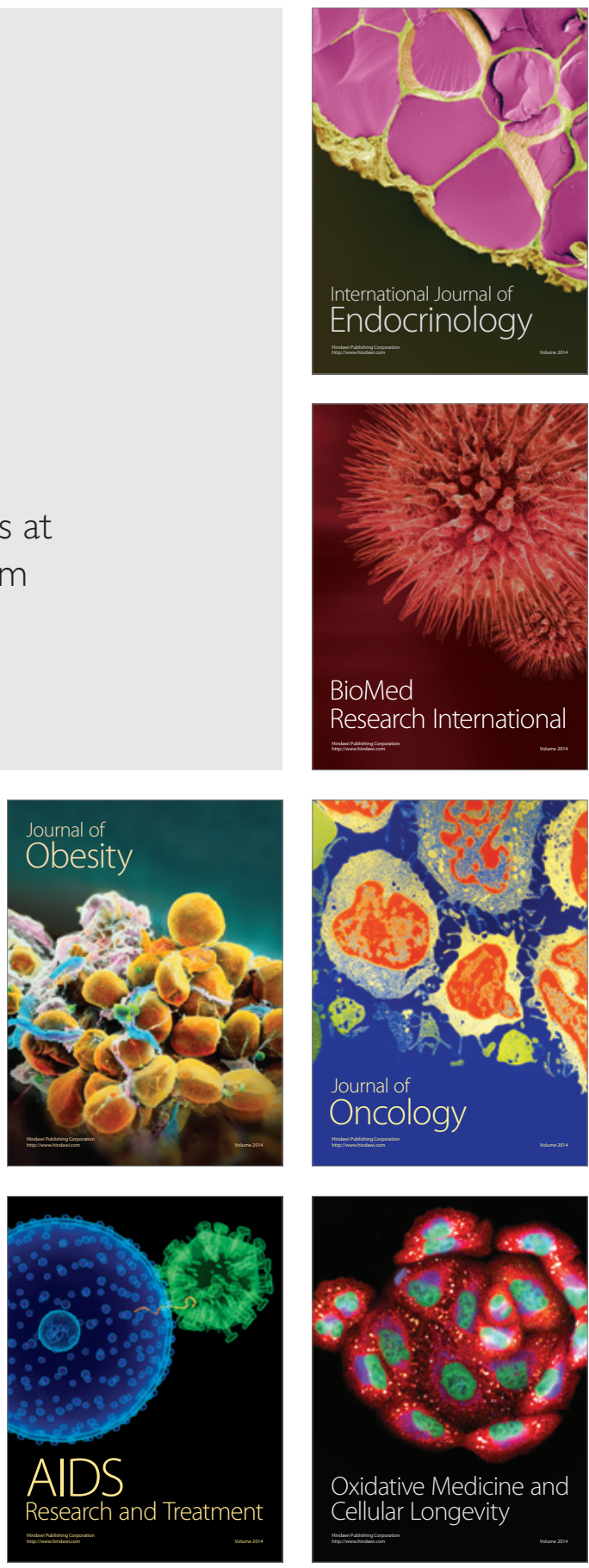